\title{
Single-Molecule Imaging of Gold-Binding Peptide Adsorbed on $\operatorname{Au}(111)$
}

\author{
Satoshi Kanata, ${ }^{*}$ Tomoaki Nishino, ${ }^{* * \dagger}$ Rie MakiUra, $* *$ Sho SaIKI, ${ }^{* * *}$ and Nobuhiko Hayashi** \\ *Department of Physics and Electronics, Graduate School of Engineering, Osaka Prefecture University, \\ 1-1 Gakuencho, Naka, Sakai, Osaka 599-8531, Japan \\ **Nanoscience and Nanotechnology Research Center, Research Organization for the 21st Century, \\ Osaka Prefecture University, 1-2 Gakuencho, Naka, Sakai, Osaka 599-8570, Japan \\ ***Department of Physical Science, Graduate School of Science, Osaka Prefecture University, 1-1 Gakuencho, \\ Naka, Sakai, Osaka 599-8531, Japan
}

\begin{abstract}
Inorganic-binding peptides, which exhibit specific binding affinity to an inorganic material, are versatile building blocks in the construction of novel bio-conjugated materials. However, very little knowledge regarding their adsorbed structures on the target material is currently available. In this article, we report on the single-molecule analysis of such polypeptides by scanning tunneling microscopy (STM). The adsorbed structure of a gold-binding peptide (GBP) on Au(111) was observed at the single-molecule level. FTIR spectroscopy revealed the helical structure of the GBP, and $a b$ initio calculations confirmed the correlation between the observed STM image and a sample helical structure. It has been demonstrated that the conformational structure of the polypeptide is highly pre-organized, allowing favorable binding onto the gold surface. Gaining such an insight into the relation between the structure and the binding function of the peptide leads to a fundamental understanding of inorganic-binding peptide, and, consequently, to a rational design of these peptides.
\end{abstract}

(Received December 27, 2012; Accepted January 21, 2013; Published April 10, 2013)

\section{Introduction}

Gaining an understanding of the interfaces between peptides and inorganic materials is a fundamental and pivotal issue in a wide range of scientific disciplines. ${ }^{1,2}$ These interfaces play central roles in, for example, blood clotting, biomineralization, and biomedical applications. Analyzing the peptide/inorganic interface, especially at the single-molecule level, remains a nontrivial challenge, primarily because of the high flexibility and chemical heterogeneity of peptides. A variety of simple polypeptides with specific binding affinity to an inorganic material have recently been developed ${ }^{3-9}$ using combinatorial selections of peptides produced through genetic-engineering techniques. ${ }^{10,11}$ Phage display and cell-surface display have been utilized to choose polypeptides that possess a high affinity for given inorganic targets. Inorganic-binding peptides were selected and identified through biopanning from random peptides displayed on the phage or cell surfaces. This biopanning process allows for the selection of a polypeptide with the desired binding affinity without knowledge about the amino acid sequence required for the binding. This is clearly an advantage of these display technologies. However, conversely, affinity selection by biopanning provides little knowledge of the suitable amino acid sequence.

Their recognition ability makes inorganic-binding peptides

$\dagger$ To whom correspondence should be addressed.

E-mail: t-nishino@21c.osakafu-u.ac.jp ideal molecular building blocks for novel nanomaterials ${ }^{12-16}$ and nanostructures. ${ }^{17-20}$ For example, it was demonstrated that a gold-binding peptide exhibits selective adsorption on $\mathrm{Au}$, even in the presence of another noble metal $(\mathrm{Pt})$ on a micropatterned surface. Kinetic analyses using a quartz crystal microbalance have revealed a higher adsorption coefficient and a lower desorption constant of this polypeptide for Au compared to Pt. ${ }^{21}$ Fusing the inorganic-binding peptide to functional biomolecules and nanomaterials provides an attractive method for the directed assembly of hybrid materials. Furthermore, the high affinity of these simple polypeptides provides a unique opportunity to investigate the interaction between the peptide and the inorganic material, which governs the peptide/inorganic material interface. Determining the molecular structure of the inorganic-binding peptide adsorbed on its target surface is important for understanding the interface. ${ }^{22-24}$

We report herein on the imaging of an individual gold-binding peptide adsorbed on a $\mathrm{Au}$ surface by scanning tunneling microscopy (STM) under ambient conditions. The adsorbed structure of the single peptide was successfully visualized. FTIR spectroscopy revealed a helical structure of the GBP, and $a b$ initio calculations confirmed the correlation between the observed STM image and the sample helical structure. Moreover, we found that peptide residues responsible for the binding affinity were aligned on the same side of the GBP helix. These results demonstrate that the conformational structure of the polypeptide is highly pre-organized, thus allowing favorable binding onto the gold surface. The results reveal that the conformational structure of the peptide is vital for binding. 


\section{Experimental}

\section{Reagents and chemicals}

The reagents were of the highest grade available. The peptide sequence, MHGKTQATSGTIQS, which exhibits binding affinity to a gold surface, ${ }^{3,21}$ was selected as the sample polypeptide. Two kinds of polypeptides were investigated in the present study: one consisting of the single gold-binding sequence (1GBP), and the other containing three repeats of the sequence (3GBP). In addition, alanine-substituted 1GBP (1GBP-Ala), in which all of the threonine residues of 1 GBP were replaced with alanine residues, was also investigated for a control experiment. All of the polypeptides were commercially synthesized (Toray Research Center, Tokyo, Japan). Deionized water purified with a Milli-Q water purification system (Japan Millipore, Tokyo, Japan) was used throughout the experiments. An atomically flat $\mathrm{Au}(111)$ thin layer, $200 \mathrm{~nm}$ thick, was prepared by thermal evaporation of $\mathrm{Au}$ (Nilaco) onto mica (Nilaco) under a vacuum. Microscope glasses $\left(10 \times 10 \mathrm{~mm}^{2}\right)$ were glued onto the $\mathrm{Au}$ layer with Epotek 377 (Epoxy Technology, Billerica, MA); these assemblies were heated at $150^{\circ} \mathrm{C}$ in an electric oven for $1.5 \mathrm{~h}$. The mica sheet was stripped off to expose an ultra-flat clean $\mathrm{Au}(111)$ surface. ${ }^{25}$ The gold-binding peptide was immediately adsorbed on the $\mathrm{Au}(111)$ surface after it had been exposed by stripping the mica. For the adsorption, aqueous solutions containing 1GBP $(40 \mu \mathrm{M})$, 1GBP-Ala $(40 \mu \mathrm{M})$, or $3 \mathrm{GBP}(13 \mu \mathrm{M})$ were applied onto the Au surface rotated at $4000 \mathrm{rpm}$ by a spin coater.

\section{Apparatus}

STM observations were carried out using a Nanoscope E (Digital Instrument, Santa Barbara, CA) at room temperature in air. The images were recorded in the constant-current mode, typically at a bias voltage of +0.50 to $+1.5 \mathrm{~V}$ and tunneling current of $0.20-0.70 \mathrm{nA}$. The FTIR spectra were obtained in vacuo at room temperature using a JASCO FTIR-6200 spectrometer with a resolution of $4 \mathrm{~cm}^{-1}$. The RAS (incident angle $=85^{\circ}$ ) measurement of the gold surface-bound 1GBP was performed withaliquid-nitrogen cooledmercury-cadmium-tellurium (MCT) detector using an untreated gold substrate as a reference.

\section{Theoretical calculations}

The initial molecular geometry of 1GBP was constructed by adjusting the backbone dihedral angles so as to form a $3_{10}$-helix according to the literature. ${ }^{26}$ This geometry was optimized at the restricted Hartree-Fock (RHF) level. An $a b$ initio molecular-orbital calculation was conducted based on the DV-X $\alpha \operatorname{method}^{27}$ using optimized molecular geometry. The square of the absolute value of the obtained wave function was then summed up from 0 to $-2.3 \mathrm{eV}$ relative to the Fermi level to estimate the local density of states (LDOS) relevant to the STM observation. Since the 1GBP molecule most probably adsorbs on Au by its aligned Ser and Thr, the molecular plane opposite to these residues was considered, whose orientation is illustrated in Fig. 4c. The LDOS was integrated with respect to the coordinate normal to this molecular plane, resulting in the theoretical STM image shown in Fig. 4d.

\section{Results and Discussion}

A peptide sequence, MHGKTQATSGTIQS, was selected as a sample polypeptide. It is known that a polypeptide containing this sequence avidly and specifically binds onto a gold surface. ${ }^{3,21}$
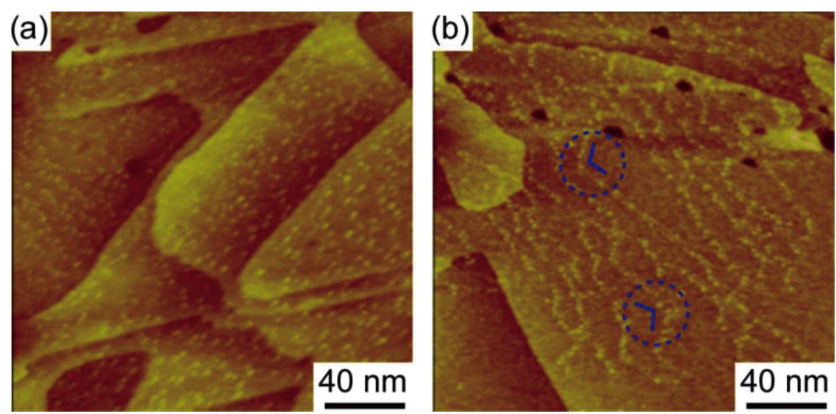

Fig. 1 Typical large-scale STM images of gold-binding peptides on $\mathrm{Au}(111)$. (a) 3GBP. Bias voltage, $0.70 \mathrm{~V}$; tunneling current, $0.70 \mathrm{nA}$. (b) 1GBP. Bias voltage, $1.1 \mathrm{~V}$; tunneling current, $0.55 \mathrm{nA}$. The characteristic bends in the peptide chains are indicated by the blue circles.

Previous studies on this gold-binding peptide revealed its binding kinetics, and an AFM observation was carried out to observe an ordered supramolecular self-assembly of a confluent peptide adlayer. ${ }^{22,28,29}$ In the present work, we observed single isolated peptides by STM. Two kinds of polypeptides were investigated in the present study: one consisting of the single gold-binding sequence (1GBP), and the other containing three repeats of the sequence (3GBP). Figures $1 \mathrm{a}$ and $1 \mathrm{~b}$ show typical large-scale STM images of the gold surface spin-coated with $3 \mathrm{GBP}$ and 1GBP solutions, respectively. Numerous protrusions were observed decorating the $\mathrm{Au}(111)$ terraces in both images. These protrusions were attributed to the polypeptides, and this interpretation was confirmed by FTIR spectroscopy (see below). The surface concentration of $3 \mathrm{GBP}$ was estimated to be $2.2 \times 10^{-12} \mathrm{~mol} / \mathrm{cm}^{2}$ based on a direct count of individual molecules in the STM image. High-resolution STM imaging revealed that some of the protrusions in the STM image of 1GBP were composed of multiple, not single, molecules. Thus, we refrain from elucidating the surface concentration of 1GBP. The most striking feature found in these images is the alignment of the sample molecules, especially apparent in Fig. 1b. The polypeptides align themselves into lines, implying anisotropic intermolecular interactions between the surface-bound peptides. In addition, there exists a characteristic bending along these lines, indicated by circles in Fig. $1 \mathrm{~b}$. The bend angle was found to be $126 \pm 12^{\circ}$. This angle correlates well with the three-fold symmetry of the $\mathrm{Au}(111)$ surface in accord with the AFM observation of the confluent monolayer of $3 \mathrm{GBP}^{29}$ The peptides were adsorbed onto the $\mathrm{Au}(111)$ surface also by simply dipping the substrate into the peptide solution. The subsequent STM imaging showed similar adsorption characterestics as observed for the sample prepared by the spin-coating procedure described above, though the dip coating caused a higher surface concentration of the peptide than the spin coating. These results indicate that peptide adsorption is in registry with the atomic arrangement of gold, and such a registry could be one of the origins of the recognition ability of the sample polypeptides.

The material selectivity of the peptide binding was elucidated at the molecular level using STM observations. First, 1GBP was applied to highly oriented pyrolytic graphite (HOPG). HOPG has been used as a substrate for peptide adsorption, and it was reported that artificial or natural polypeptides formed closely packed $\beta$-sheets. ${ }^{30}$ No protrusions, as seen on the gold surface, were observed (Fig. 2a). However, a few large aggregates were found on the terrace. No similar structure was found on unmodified clean HOPG surfaces (Fig. S1, Supporting 

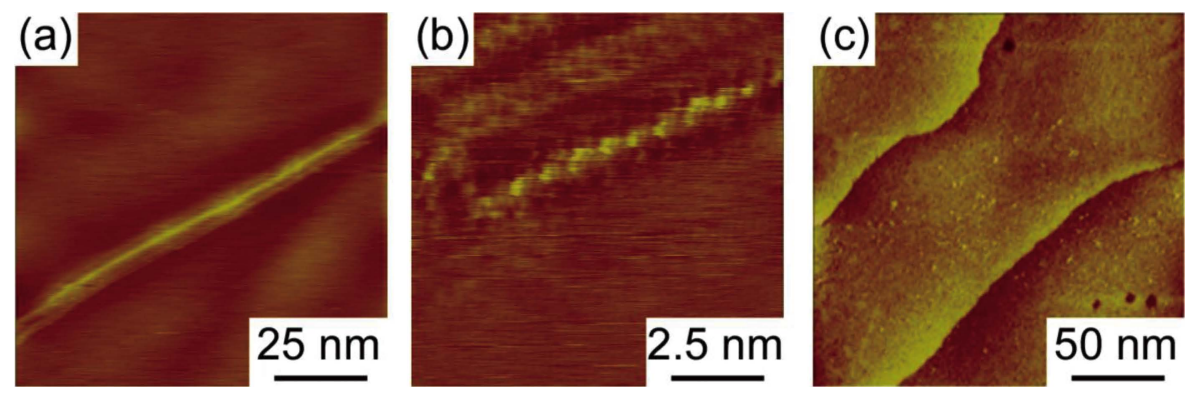

Fig. 2 STM images of $1 \mathrm{GBP}$ on HOPG. Bias voltage, (a) 0.60 and (b) $0.70 \mathrm{~V}$; tunneling current, (a) 0.72 and (b) $0.60 \mathrm{nA}$. (c) STM image of $1 \mathrm{GBP}$ on $\mathrm{I} / \mathrm{Au}(111)$. Bias voltage, $1.3 \mathrm{~V}$; tunneling current, $0.55 \mathrm{nA}$.

Information). High-resolution STM images showed that parallel-oriented peptides constitute such aggregates (Fig. 2b). Similar results were obtained with 3 GBP on HOPG. A gold surface modified with iodine ${ }^{31}$ was also used to investigate the selectivity of the gold-binding peptides. Figure $2 \mathrm{c}$ shows a representative STM image of the I/Au(111) substrate after the modification process using the 1GBP solution. In contrast to the bare $\mathrm{Au}(111)$ surface (Fig. 1), only sparse adsorbates existed on the $\mathrm{I} / \mathrm{Au}(111)$ surface. Similarly, only a negligible amount of $3 \mathrm{GBP}$ was found on $\mathrm{I} / \mathrm{Au}(111)$. Control experiments using alanine-substituted 1GBP also exhibited a decreased binding affinity of the polypeptide to the $\mathrm{Au}(111)$ surface (see below). These results demonstrate the stringent selectivity of the gold-binding polypeptides.

FTIR spectroscopy was employed for the characterization of 1GBP on the $\mathrm{Au}(111)$ surface. The measured reflection-absorption spectrum (RAS) is shown in Fig. 3b, together with that of 1GBP powder (Fig. 3a). The absorptions around $1700-1600 \mathrm{~cm}^{-1}$ and at $1525 \mathrm{~cm}^{-1}$ in the $1 \mathrm{GBP}$ powder spectrum are assigned to the amide I ( $\mathrm{C}=\mathrm{O}$ stretching) and amide II ( $\mathrm{N}-\mathrm{H}$ bending) vibrational modes, respectively. ${ }^{32}$ The most prominent band in the amide I region (Fig. 3a) occurs at $1623 \mathrm{~cm}^{-1}$, and it can be attributed to intermolecular $\beta$-sheet structures; this assignment is supported by the presence of an amide II band at $1525 \mathrm{~cm}^{-1} .^{33}$ At the same time, the observation of a band at $1697 \mathrm{~cm}^{-1}$ together with the shoulder evident at $1540 \mathrm{~cm}^{-1}$ is consistent with the existence of intramolecular $\beta$-sheet structures in the $1 \mathrm{GBP}$ bulk powder. ${ }^{33}$ Finally, the peak present at $1667 \mathrm{~cm}^{-1}$ in the amide I region and the shoulder at around $1550 \mathrm{~cm}^{-1}$ (amide II region) reveal the presence of $3_{10}$-helices in the bulk sample. ${ }^{34,35}$ As shown in Fig. 3b, the amide I and II bands also clearly appear in the RAS spectrum of the Au(111) surface spin-coated with 1GBP, demonstrating the successful attachment of the polypeptides to the gold surface. This also confirms that the protrusions observed in the STM images (Fig. 1) correspond to gold-binding peptides. At the same time, however, significant differences between the spectra of the powder and the monolayer adsorbed onto $\mathrm{Au}(111)$ are evident in both spectral regions. Very importantly, the complete disappearance of the band at $1623 \mathrm{~cm}^{-1}$ provides strong evidence that the 1GBP monolayer on $\mathrm{Au}(111)$ does not include any intermolecular $\beta$-sheet structures. This supports the absence of aggregation occurring in the surface-bound 1GBP and is consistent with the alignment of the peptide molecules on the gold surface as seen in the STM images (Fig. 1b). In contrast, the band centered at $1667 \mathrm{~cm}^{-1}$ remains prominent and is accompanied by the band at $1550 \mathrm{~cm}^{-1}$,

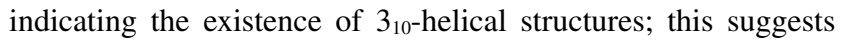
either selective binding of the $3_{10}$-helical structures in the 1 GBP

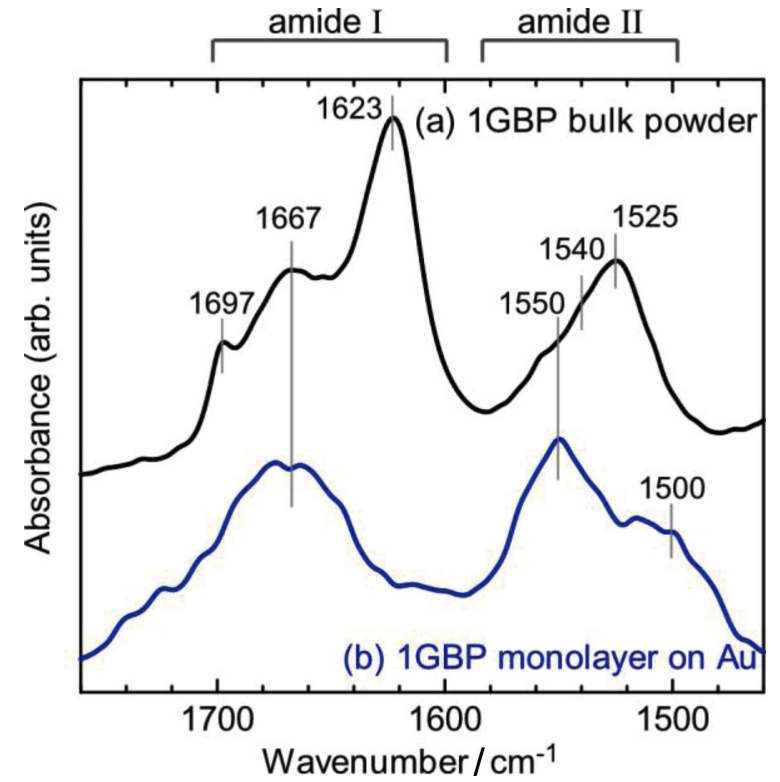

Fig. 3 Amide I and II band regions of the FTIR spectra of 1GBP. (a) Bulk powder and (b) a monolayer bound to the $\mathrm{Au}(111)$ surface.

to the gold surface, or a structural transformation from the $\beta$-sheet to the $3_{10}$-helix occurring upon adsorption. Furthermore, the shoulder appearing in the low-frequency side of the amide II region $\left(\sim 1500 \mathrm{~cm}^{-1}\right)$ can be attributed to $\mathrm{N}-\mathrm{H}$ groups without hydrogen bonds, while the prominent band at $1550 \mathrm{~cm}^{-1}$ represents hydrogen-bonded amide II units; the amide II mode is known to show a blue shift when the $\mathrm{N}-\mathrm{H}$ group is hydrogen-bonded. ${ }^{36}$ The hydrogen-bond free N-H parts clearly seen in the 1GBP bound to the gold substrate may effectively interact with the gold surface and play an important role in the selective binding properties.

Further STM imaging was performed to investigate the binding structure at the single-molecule level. Figure 4a presents an STM image of a single 3GBP molecule that appeared as an oval-shaped protrusion. Although the $\beta$-sheet structure was anticipated according to the literature, ${ }^{37}$ no such structure could be discerned in the STM image of Fig. 4a, but a faint elliptical bright contrast could be seen. This result was attributed to a deteriorated spatial resolution owing to hydrated water molecules. Since water molecules possess a high conductivity that gives rise to an enhanced contrast in STM images, hydrated water molecules can smear out intramolecular specimen structures. In addition, the structural flexibility of the sample 

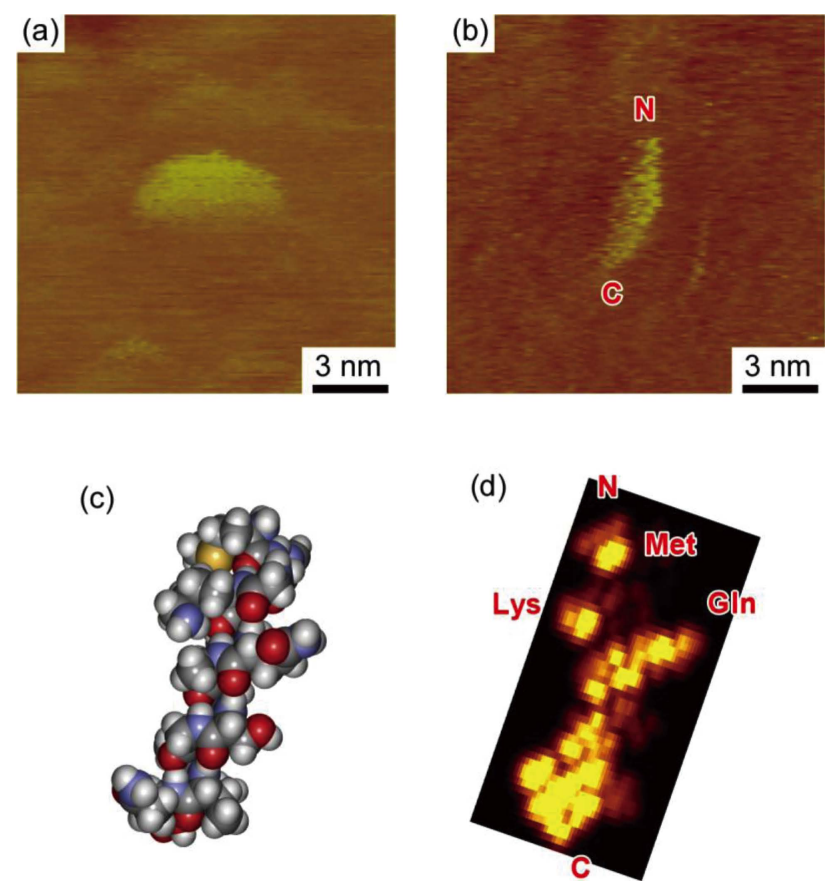

Fig. 4 High-resolution STM images of the gold-binding peptides on $\mathrm{Au}(111)$. (a) 3GBP: bias voltage, $1.1 \mathrm{~V}$; tunneling current, $0.60 \mathrm{nA}$. (b) 1GBP: bias voltage, $0.90 \mathrm{~V}$; tunneling current, $0.60 \mathrm{nA}$. (c) Molecular structure of $1 \mathrm{GBP}$ in a $33_{10}$-helix conformation optimized at the RHF level. (d) LDOS image of $1 \mathrm{GBP}$ calculated for the molecular conformation in (c). $\mathrm{N}$ and $\mathrm{C}$ represent the $\mathrm{N}$ - and C-termini of $1 \mathrm{GBP}$, respectively.

molecules can degrade the resolution by thermal fluctuation. Considering that the $\beta$-sheet structures of 3GBP are formed by three 1GBP strands, 1GBP is less hydrated and more rigid than 3GBP owing to the decreased molecular dimension. Indeed, 1GBP allowed high-resolution imaging of its molecular structure adsorbed on $\mathrm{Au}(111)$, as shown in Fig. 4b. The bent structure of the single 1GBP molecule was clearly observed in the STM image. Although submolecularly resolved STM images have been reported for linear hydrocarbon molecules, ${ }^{38}$ similar resolution is hardly achieved for biopolymers, including proteins, mainly because of their conformational flexibility. The spatial resolution of Fig. $4 \mathrm{~b}$ is comparable to that of the recently reported STM image of cytochrome c. ${ }^{39}$ The cytochrome $c$ was carefully adsorbed on metal surfaces by electrospray ion beam deposition, and STM imaging was conducted in an ultrahigh vacuum condition. In the present study, the sample peptide was adsorbed simply by spin-coating and observed in an ambient condition; nevertheless, the similar resolution was achieved for $1 \mathrm{GBP}$. We ascribe this result to the strong binding affinity of the gold binding peptide, leading to the firm immobilization ideal for the STM imaging.

The single-molecule structure experimentally observed on the gold surface was compared with the theoretically predicted gas-phase structure in order to determine whether structural changes occur upon the adsorption of the gold-binding peptide on its binding target. According to the FTIR measurements described above, the surface-bound 1GBP adopts the $3_{10}$-helical conformation. The initial $33_{10}$-helix structure of the 1GBP molecule was optimized at the restricted Hartree-Fock (RHF) level. ${ }^{26}$ GBP retained the same helical structure after geometry optimization (Fig. 4c). It was found that four out of five serine or threonine residues of $1 \mathrm{GBP}$ were located in line on the same side of the helix in the optimized structure (see Fig. S2, Supporting Information). It has been suggested that these polar amino acids account for the binding of the polypeptide on gold ${ }^{37}$ therefore, the aligned serine and threonine residues most probably constitute the binding sites on the gold surface. For this binding orientation, the local density of states (LDOS) near the Fermi level, which makes a dominant contribution to an STM image (Fig. 4d), was calculated. The LDOS map exhibited higher intensities for C-terminal sequences (labeled " $\mathrm{C}$ " in Fig. 4d), including serine, glutamine, and threonine, in addition to methionine, lysine, and glutamine near the $\mathrm{N}$ terminal ("Met," "Lys," and "Gln" in Fig. 4d, respectively). Overall, the LDOS map for the 1GBP molecule showed a bent structure, consistent with the observed STM image. A slight deviation between the calculated LDOS and the observed STM image was noted for the "Gln" protrusion, which is apparent in Fig. 4d, but absent in Fig. 4b. This is attributed to the rotation of the long side chain of the glutamine residue. These results provide an insight into the role of the secondary structure in 1GBP adsorption. The STM image of the single 1GBP molecule in Fig. $4 \mathrm{~b}$ agrees well with the LDOS image calculated on the basis of the optimized $3_{10}$-helix structure in the gas phase. By adopting this structure, most of the serine and threonine residues, which lead to the binding affinity, ${ }^{37}$ in the $1 \mathrm{GBP}$ sequence are found to be on the same side of the helix.

Control experiments were carried out to confirm the role of these amino-acid residues in the peptide adsorption. All of the threonine residues of $1 \mathrm{GBP}$ were replaced with alanine residues, and this alanine-substituted 1GBP (1GBP-Ala) was observed with STM. In stark contrast to the unsubstituted 1GBP, STM image of 1GBP-Ala showed no distinct contrast of the adsorbate, and only fuzzy bumps were observed (see Fig. S3, Supporting Information). Given nearly the same molecular dimension of the 1GBP and 1GBP-Ala, the obscure appearance of the 1GBP-Ala is most probably due to a sizable surface mobility of this peptide, because such observations were frequently reported for mobile adsorbates. ${ }^{40}$ The surface mobility indicates a decreased binding affinity caused by the alanine substitution. These results lead to the conclusion that the conformational structure of 1GBP polypeptide is highly pre-organized, allowing favorable binding to the gold surface. Although the deliberate design of simple peptides that show selective binding to inorganic materials was achieved by engineering only the amino acid sequences, ${ }^{41-43}$ our conclusion suggests that the higher order structure of the peptide also needs to be taken into account to achieve a sophisticated binding affinity and selectivity.

\section{Conclusions}

In summary, we report on the single-molecule imaging of a gold-binding peptide. STM observations, in combination with FTIR spectroscopy, and theoretical calculations reveal that the conformational structure of the polypeptide contributes to its metal-binding affinity. Gaining such an insight into the correlation between the structure and the binding function of the peptide leads to a fundamental understanding of inorganic-binding peptides and, consequently, to the rational design of these peptides to create novel bio-nano conjugated materials. It remains difficult to characterize biomolecules on a solid surface at the single-molecule level because of the structurally and chemically complex nature of the analytes. Despite this difficulty, as shown in the present study, combining STM observations with theoretical simulations along with spectroscopic measurements offers an effective means for such 
analyses. Further details of the adsorbed structure of the gold-binding peptide can be obtained from molecular STM tips, which enable chemically selective imaging. ${ }^{44,45}$ Such experiments are currently in progress in our laboratory.

\section{Acknowledgements}

This work was supported by Special Coordination Funds for Promoting Science and Technology commissioned by MEXT of Japan, and by JSPS KAKENHI (23550103).

\section{Supporting Information}

STM images of unmodified HOPG surface, alignment of serine and threonine residues in 1GBP, and STM image of alanine-substituted 1GBP. This material is available free of charge on the Web at http://www.jsac.or.jp/analsci/.

\section{References}

1. C. A. Mirkin and T. A. Taton, Nature, 2000, 405, 626.

2. S. Zhang, Nat. Biotechnol., 2003, 21, 1171.

3. S. Brown, Nat. Biotechnol., 1997, 15, 269.

4. M. Sarikaya, C. Tamerler, A. K. Y. Jen, K. Schulten, and F. Baneyx, Nat. Mater., 2003, 2, 577.

5. S. Brown, M. Sarikaya, and E. Johnson, J. Mol. Biol., 2000, 299, 725.

6. A. K. Sinensky and A. M. Belcher, Adv. Mater., 2006, 18, 991.

7. S. R. Whaley, D. S. English, E. L. Hu, P. F. Barbara, and A. M. Belcher, Nature, 2000, 405, 665.

8. F. Baneyx and D. T. Schwartz, Curr. Opin. Biotechnol., 2007, 18, 312 .

9. R. R. Naik, S. J. Stringer, G. Agarwal, S. E. Jones, and M. O. Stone, Nat. Mater., 2002, 1, 169.

10. P. J. Yoo, K. T. Nam, J. F. Qi, S. K. Lee, J. Park, A. M. Belcher, and P. T. Hammond, Nat. Mater., 2006, 5, 234.

11. M. Fischlechner, L. Toellner, P. Messner, R. Grabherr, and E. Donath, Angew. Chem., Int. Ed., 2006, 45, 784.

12. K. T. Nam, D. W. Kim, P. J. Yoo, C. Y. Chiang, N. Meethong, P. T. Hammond, Y. M. Chiang, and A. M. Belcher, Science, 2006, 312, 885.

13. Y. J. Lee, H. Yi, W. J. Kim, K. Kang, D. S. Yun, M. S. Strano, G. Ceder, and A. M. Belcher, Science, 2009, 324, 1051.

14. C. B. Mao, C. E. Flynn, A. Hayhurst, R. Sweeney, J. F. Qi, G. Georgiou, B. Iverson, and A. M. Belcher, Proc. Natl. Acad. Sci. U. S. A., 2003, 100, 6946.

15. R. M. Kramer, C. Li, D. C. Carter, M. O. Stone, and R. R. Naik, J. Am. Chem. Soc., 2004, 126, 13282.

16. M. J. Pender, L. A. Sowards, J. D. Hartgerink, M. O. Stone, and R. R. Naik, Nano Lett., 2006, 6, 40.

17. T. Kacar, J. Ray, M. Gungormus, E. E. Oren, C. Tamerler, and M. Sarikaya, Adv. Mater., 2009, 21, 295.

18. J. H. Wei, T. Kacar, C. Tamerler, M. Sarikaya, and D. S. Ginger, Small, 2009, 5, 689.
19. K. Sano, S. Yoshii, I. Yamashita, and K. Shiba, Nano Lett., 2007, 7, 3200.

20. K. Sano, H. Sasaki, and K. Shiba, J. Am. Chem. Soc., 2006, $128,1717$.

21. C. Tamerler, M. Duman, E. E. Oren, M. Gungormus, X. Xiong, T. Kacar, B. A. Parviz, and M. Sarikaya, Small, 2006, 2, 1372.

22. C. R. So, C. Tamerler, and M. Sarikaya, Angew. Chem., Int. Ed., 2009, 48, 5174.

23. T. Hayashi, K. Sano, K. Shiba, Y. Kumashiro, K. Iwahori, I. Yamashita, and M. Hara, Nano Lett., 2006, 6, 515.

24. E. E. Oren, C. Tamerler, and M. Sarikaya, Nano Lett., 2005, $5,415$.

25. P. Wagner, M. Hegner, H. J. Guntherodt, and G. Semenza, Langmuir, 1995, 11, 3867.

26. I. A. Topol, S. K. Burt, E. Deretey, T. H. Tang, A. Perczel, A. Rashin, and I. G. Csizmadia, J. Am. Chem. Soc., 2001, 123, 6054 .

27. H. Adachi, M. Tsukada, and C. Satoko, J. Phys. Soc. Jpn., 1978, $45,875$.

28. C. Tamerler, E. E. Oren, M. Duman, E. Venkatasubramanian, and M. Sarikaya, Langmuir, 2006, 22, 7712.

29. C. R. So, J. L. Kulp, E. E. Oren, H. Zareie, C. Tamerler, J. S. Evans, and M. Sarikaya, ACS Nano, 2009, 3, 1525.

30. L. Liu, K. Busuttil, S. Zhang, Y. Yang, C. Wang, F. Besenbacher, and M. Dong, Phys. Chem. Chem. Phys., 2011, 13, 17435.

31. M. Kunitake, N. Batina, and K. Itaya, Langmuir, 1995, 11, 2337.

32. P. I. Haris and D. Chapman, Biopolymers, 1995, 37, 251.

33. M. Di Foggia, P. Taddei, C. Fagnano, A. Torreggiani, M. Dettin, S. Sanchez-Cortes, and A. Tinti, J. Mol. Struct., 2009, 924 - 926, 120.

34. M. Boncheva and H. Vogel, Biophys. J., 1997, 73, 1056.

35. A. E. Strong and B. D. Moore, J. Mater. Chem., 1999, 9, 1097.

36. H. Maekawa, M. De Poli, C. Toniolo, and N. H. Ge, J. Am. Chem. Soc., 2009, 131, 2042.

37. R. Braun, M. Sarikaya, and K. Schulten, J. Biomater. Sci., Polym. Ed., 2002, 13, 747.

38. S. De Feyter and F. C. De Schryver, J. Phys. Chem. B, 2005, 109, 4290 .

39. Z. Deng, N. Thontasen, N. Malinowski, G. Rinke, L. Harnau, S. Rauschenbach, and K. Kern, Nano Lett., 2012 , 12, 2452.

40. A. E. Baber, H. L. Tierney, and E. C. H. Sykes, ACS Nano, 2008, 2, 2385.

41. B. R. Peelle, E. M. Krauland, K. D. Wittrup, and A. M. Belcher, Langmuir, 2005, 21, 6929.

42. R. L. Willett, K. W. Baldwin, K. W. West, and L. N. Pfeiffer, Proc. Natl. Acad. Sci. U. S. A., 2005, 102, 7817.

43. R. Levy, N. T. K. Thanh, R. C. Doty, I. Hussain, R. J. Nichols, D. J. Schiffrin, M. Brust, and D. G. Fernig, J. Am. Chem. Soc., 2004, 126, 10076.

44. T. Nishino, T. Ito, and Y. Umezawa, Proc. Natl. Acad. Sci. U. S. A., 2005, 102, 5659.

45. T. Nishino, S. Kanata, and Y. Umezawa, Chem. Commun., 2011, 47, 7467. 